\title{
PARAMETERIZED COMPUTER AIDED DESIGN OF STUBBLE CLEANER
}

\author{
Lige Wen ${ }^{1,2}$, Jianqiao $\mathrm{Li}^{1, *}$, Xiuzhi Zhang ${ }^{2}$, Benard Chirende ${ }^{1}$ \\ ${ }^{I}$ The Key Laboratory for Terrain-Machine Bionics Engineering, Ministry of Education, Jilin \\ University, Changchun, China, 130025 \\ ${ }^{2}$ College of Mechanical Science and Engineering, Jilin University, Changchun, China, 130025 \\ * Corresponding author, Address: No. 5988, Renmin Street, Changchun, P. R. China, 130025, \\ Tel: +86-431-85095760-8407, Fax:+86-431-85095575, Email: jqli@jlu.edu.cn
}

\begin{abstract}
The traditional agricultural machine design were represented as $2 \mathrm{D}$ drawing, which is difficult to modify, and also not intuitive in the solid way for the form and structure. Therefore, software, UG-NX3, was used to conduct parameterized design of stubble cleaner parts. The parts were designed associatively and assembled virtually. The structure of the whole machine and the spatial distribution of parts can be seen and analyzed intuitively. Under UG-NX3 circumstance, the designed 3D model can be transformed automatically to $2 \mathrm{D}$ drafting which is used in fabrication and production. The result proved that computer aided parameterized design can allow dynamical operation, preview and repeated modification of the design, reliably and quickly. Therefore, the optimum design efficiency of stubble cleaner is improved; 3D modeling time and 2D drafting time is greatly decreased.
\end{abstract}

Keywords: Stubble cleaner, computer aided design, parameterized design

\section{INTRODUCTION}

In recent years, with the rapid development of computer technology, 3D design and virtual assembling technology were introduced into the mechanical design field, and as a result, product update frequency and design efficiency were greatly increased (Wen, 2003). These machines are also gradually incorporated into the agricultural machine design field (Yang

Wen, L., Li, J., Zhang, X. and Chirende, B., 2008, in IFIP International Federation for Information Processing, Volume 258; Computer and Computing Technologies in Agriculture, Vol. 1; Daoliang Li; (Boston: Springer), pp. 11-18. 
et al., 2004; Yang et al., 2002; Yuan et al., 2006). Computer aided design will be the necessary trend of agriculture machine design field (Yan et al., 2004). Prior to using the virtual assembling technology, design for stubble cleaner was mainly $2 \mathrm{D}$ design in which the structure of parts and the whole machine could not be easily visualized, hence associative relationship among parts could be hardily established. In addition, parts assembly and interference checkup could not be conducted. In order to resolve the above problems, parameterized design and virtual assembly were used to design stubble machines based on UG-NX3 (Fu, 2005; Zhao et al., 2005), and this is in line with the trend of stubble cleaner development (Wu et al., 2000).

\section{PARAMETERIZED DESIGN AND ASSOCIATIVE DESIGN}

\subsection{Parameterized design}

Parameterized design method is a new kind of 3D design method, and UG- NX3 is one of the representational 3D parameterized design software. There is driving parameter and calculation parameter under the environment of UG-NX3. Driving parameter means that a variable can be evaluated and its value can be changed at will, whilst calculation parameter is the parameter obtained through calculation based on driving parameter. UGNX3 has strong sketch functions, utilizing dimension constraint and geometry constraint to drive the sketch, dimensions and shapes of the sketch alter with respect to the change of constraints. Design modification can be done repeatedly and quickly, and the time spent on 3D modeling is markedly reduced.

\subsection{Associative design}

Associative design means setting up associative relationship among parts such that the associative parts change their sizes and structures simultaneously. Associative design method avoids interference among parts, and makes modifying work convenient and accurate. UG-NX3 provides two assembly methods, one is Bottom-Up design method and the other is TopDown design method. The former first establishes models of all parts, and then assembles them. Associative constraint must be set up. In contrast, the latter sets up main parts first and other parts come into being according to 
their associative relationship with the main parts and the dimensions of the main parts. The latter suits people's design habit very well and avoids assembling interposition, therefore improving design efficiency. WAVE Geometry Linker in UG-NX3 serves the Top-Down design method. Parameter modeling establishes interrelated relationships within a part, while WAVE Geometry Linker extends this notion to set up associative relationship among different parts.

\section{PARAMETERIZED DESIGN FOR PARTS OF STUBBLE CLEANER}

\subsection{Parts of stubble cleaner}

The main function of stubble cleaner is cutting the crop stubble into small pieces and mixing them with earth uniformly and rotarily ridging the earth. Stubble cleaner mainly consists of four components.

$\diamond$ Frame, which supports the whole machine and joins with power framework, and other parts assembled on it.

$\diamond$ Gear-box, which changes the power transmission direction and speed of motion, it is composed of gears with straight tooth, transmission shaft and axletree seat.

$\diamond$ Stubble roller, which cuts up the stubble and ploughs up the earth, it consists of blade tray, stubble-cutting blade, square shaft and axletree seat.

$\diamond$ Shield, whose function is to break up the earth block, ridge the earth, and mix the earth and the stubble.

Next step is to determine main parts of every component, and to make clear the associative relationship among parts.

\section{$3.2 \quad 3 D$ design of parts}

Part modeling process is as shown in Figure 1. Firstly, the main part of every component is chosen; next, structure is analyzed, sketch is constructed, driving dimension and calculating dimension are given; then associative design to other dimensions is conducted and then feature instantiation is ensured; finally, feature-based 3D parameterized model of parts of stubble cleaner can be obtained. 


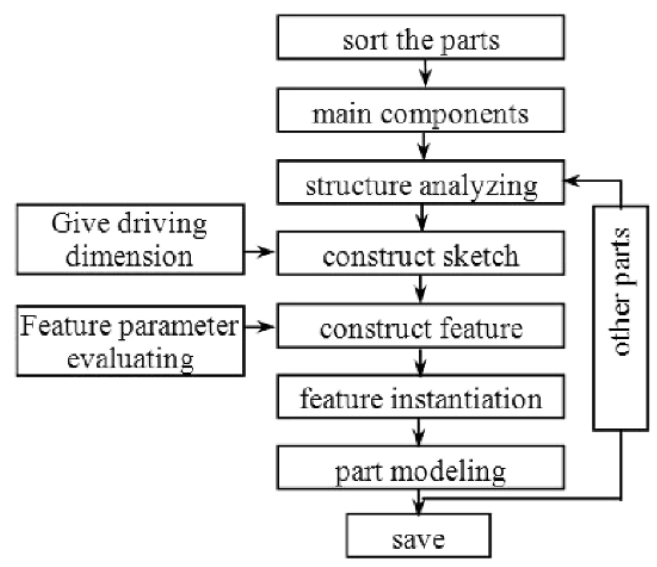

Figure 1. Modeling process for parts

\subsubsection{Parameterized design of spur gear in gear-box}

There is one pair of bevel gears and two pairs of spur gears in the cleaner. One of their 3D models is shown in Figure 2. The two bevel gears which are used to change the direction of rotating speed have the same tooth number, and their sizes remain constant. Spur gears are used to change rotating speed, because stubble cleaners are usually designed to operate at different rotating speeds.

The course of parameterized design of spur gears is as follows. Driving parameter of the gear is composed of pressure angle, modulus, tooth number, while calculation parameter consists of addendum circle diameter, gear root diameter, graduated circle diameter and base circle diameter etc.. When modifying driving parameter, the structure and size of the gear change correspondingly. Figure 3 shows that when gear tooth number changes from 19 to 24 , the structure of the gear is also altered. Associative relationship is set up between assembly hole diameter of the gear and shaft diameter.

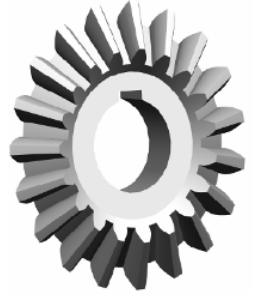

Figure 2. Straight bevel gear of stubble cleaner

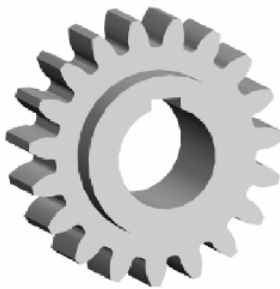

Gear tooth number 19

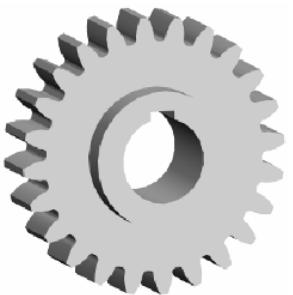

Gear tooth number 24

Figure 3. Changing of gear structure 


\subsubsection{Design of stubble blade and blade tray}

The main components in stubble roller are left blade \& right blade and four blade trays assembled at different angles. 3D model of stubble blade and one of the blade trays are as shown in Figure 4. Respective assembling holes in blade tray and stubble blade are associative, when the dimension of the assembling hole in blade changes, that of blade tray changes accordingly. The dimensions of the center square holes of the stubble trays are related to the dimensions of the square shaft.
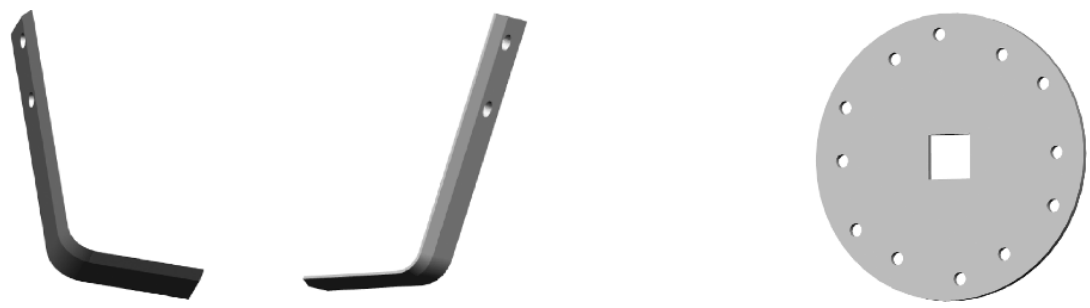

Figure 4. Stubble blades and one of the blade trays

\subsubsection{Design of cushion Using WAVE Geometry Linker}

Modeling method of axletree seat cushion is utilized as an example in order to introduce WAVE Geometry Linker design method, which is a TopDown assembly method. The process of modeling axletree seat cushion using WAVE Geometry Linker is as follows:

$\diamond$ Open "assemblies" module under UG-NX3 environment, then set up a new file; choose Non-Master Part in "New Part File" dialog box. UG-NX3 provides a function of Master Part, the formerly established model such as axletree seat is set as a Master Part, the dimensions of which can be modified, resulting in that of Non-Master Part changing accordingly. Dimensions cannot be directly changed on the Non-Master Part.

$\diamond$ Use "Add Existing" command to recall the existing axletree seat, then making use of the command "WAVE Geometry Linker", choosing assembling surface of the axletree seat where the cushion will be assembled. Now, the surface feature can be obtained, and then using Pad command, giving the thickness, the cushion is obtained as in Figure 5.

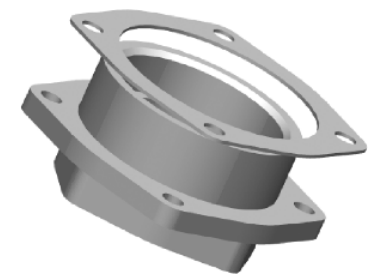

Figure 5. Create cushion using WAVE 
Finally, the dimension associative relationship between cushion and axletree seat is established.

\section{VIRTUAL ASSEMBLY}

The stubble roller was virtually assembled by 3D models designed above firstly. Then it together with other main components and minor parts was virtually assembled making use of Wave commands to make integrated virtual stubble cleaner.

\subsection{Virtual assembling of stubble roller}

Center command was used in Assemblies module of UG-NX3 to assemble square shaft and stubble trays, align the four stubble trays to the square shaft; then adapt the distance between every two trays and the distances between the outboard tray and the square shaft end should be same. Next, assemble one blade on a stubble tray with Mate command and Align command and assemble other 5 blades on the stubble tray using Component Arrays command. Utilize the same method to assemble 3, 3 and 6 blades respectively on the other 3 stubble trays and they make a total of 18 blades, 6 blades on the outboard two trays respectively and 3 ones on the inboard two trays respectively. Then we get a stubble roller set as Figure 6 shows. The 18 blades were arranged helically and have a $20^{\circ}$ gap when cutting into the earth. It is seen clearly at different direction in Figure 6 that there is no interference in the assemble body.

\subsection{Virtual assembling of the whole stubble cleaner}

Figure 7 is 3D assembly model of the whole stubble cleaner in which the shield board of the left roller, one of the shield boards of the right roller and the side shield boards are hidden. The structure of the whole machine and the relationship among parts can be seen intuitively. Under the assembly module, interference among parts can be checked up, and the gap between parts can be examined. If unreasonable condition exists, then it is necessary to return the Master Part for modification. Repeat the process until the assembly is dead-on. Using the above assembly method can minimize errors from unreasonable assembly. 


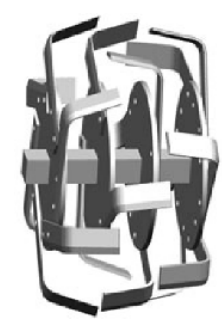

Figure 6. Virtually assemble the stubble roller

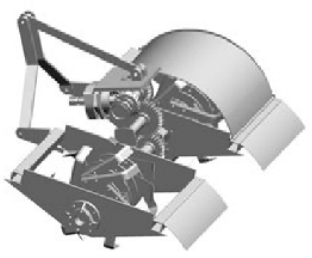

Figure 7. 3D modeling of stubble cleaner

\section{DRAFT CREATION}

UG-NX3 has the function to transform 3D module to 2D drafting automatically, which is required in producing and processing. Different kinds of views can be obtained under UG-NX3 circumstance, such as sections, axonometric drawing, partial enlarged view, and so on. Dimensions and shape, location tolerance and roughness can be given to drafting here. Drafting can be output as a file in kinds of formats, in which *.dxf file and *.dwg file can be read by AutoCAD. 3D model and 2D drafting are structure and size interrelated, when the 3D model is modified 2D drafting changes accordingly. Therefore the data consistency of $3 \mathrm{D}$ entity and $2 \mathrm{D}$ drafting is ensured.

\section{CONCLUSIONS}

$\diamond$ Parts and components of stubble cleaner were parameterized designed using UG-NX3, relationship between parts and a part lib was built up. When the size of a part is changed, that of the relational parts will be changed correspondingly, which makes it convenient to change the measurement and the profile when needed later.

$\diamond$ Conducting parameterized design, associative design and virtual assembly, and gap and interference checkup to stubble cleaner parts under UG-NX3 environment makes product design more intuitional. It improves the efficiency of remodel design and serial design of stubble cleaner, and ensures high design precision.

$\diamond$ Using this method, 2D drafting of stubble cleaner and parts can be gained conveniently and speedily. And the presented method realizes associative modification and ensures data consistency of 3D entity and 2D drawing. 
$\diamond$ The method used here would provide a reference and template for other agricultural framework design using UG-NX3 software.

\section{ACKNOWLEDGEMENTS}

The authors would like to acknowledge the referees for their helpful comments to improve the presentation of this paper. The item is supported by the National Natural Science Foundation of China (Grant No.50635030) and Projection of Science Committee of Jilin Province, China (Item No. 20050539).

\section{REFERENCES}

$\mathrm{Fu}$ Benguo, editor in chief, UG NX 3.0 3D Mechanical Design, Publishing House of Mechanical Industry, Beijing, China: 2005

Translate and edit: Zhao Bo, Zhang Qin, UG-NX2 Correlation Parameterized Design Training Tutorial, Press of Tsing Hua University, Beijing, China: 2005

Wen Bangchun, Zhou Zhicheng, Han Qingkai, et al., Important Role of Products Design of Modern Machinery in the Research and Development of New Products-Study on the Threefold Method "Dynamic Design, Intelligent Design and Partial Virtual Design" Face to Products Generalization Quality, Chinese Journal of Mechanical Engineering, 2003, 39(10): 43-52

Wu Zi-yue, Gao Huan-wen. Present state and development on technology of stubble chopping, Journal of China Agricultural University, 2000, 5(4): 46-49

Yan Chu-liang, Yang Fang-fei, Zhang Shu-ming, Digitized Design Technology and its Application in Agricultural Machinery Design, Transactions of Chinese Society of Agricultural Machinery, 2004, 35(6): 211-214

Yang Fu-zeng, Fu Xiang-hua, Yang Fang, et al., Research on Parametric Modeling of Parts of Seeding Machine Based on Pro/E, Transactions of Chinese Society of Agricultural Machinery, 2002, 33(4): 66-68

Yang Xin, liu Jun-feng, Feng Xiao-jing, Feature Modeling and Assembling Conjunction Design on Wheat Precision Seedmeter, Transactions of The Chinese Society of Agricultural Enginneering, 2004, 20(3): 89-92

Yuan Rui, Ma Xu, Ma Cheng-lin, et al., Virtual manufacturing and motion simulation of precision planter unit, Journal of Jilin University (Engineering and Technology Edition), 2006, 36(4): 523-528 